\title{
Information System of Scenario Strategic Planning
}

\author{
Denis R. Tenchurin \\ State University - Higher School of Economics, Moscow, Russia \\ dtenchurin@gmail.com \\ Maxim P. Shatilov \\ State University - Higher School of Economics, Moscow, Russia \\ maxim.shatilov@gmail.com
}

Scientific advisor: prof. Sergei M. Avdoshin

State University - Higher School of Economics, Moscow, Russia

savdoshin@hse.ru

\begin{abstract}
This paper gives an overview of the concept of a new system to support decision-making process in the area of strategic management company - DEM. To ensure that in the modern world the company remains leader in its industry it needs to continually adjust its development strategy to changes. Nevertheless, there is no unified methodological framework for strategic planning. Also there are no strategic planning systems to help managers make strategic decisions (a problem of choice of the one of the development strategies). Software Engineering Department Higher School of Economics, in collaboration with the EPAM company, conducted research in this area; the interim results have been examined in this work.
\end{abstract}

\section{Introduction}

Today modern fast-changing market of goods and services is forcing companies to review their strategic decisions and develop new ones. In order to succeed companies should comprehensively evaluate the existing internal problems, and problems that may arise in the future. Moreover, they should be able to predict the impact of external factors on internal business processes, as well as the behavior of competitors and possible changes in the environment.

Based on the analysis of the strengths and weaknesses of contemporary strategic planning methodologies the new concept and its software prototype - Dynamic Enterprise Management (DEM) were developed. The research was conducted within Software Engineering Department of Higher School of Economics in collaboration with EPAM Systems. DEM makes it possible to find the optimum of strategic development plan provided by the company.
Thus with the right mix of resources the company can succeed.

As has been proved in practice, even a plenty of detailed information doesn't help managers to make, and the main thing, to implement strategic decisions. Thus the concept of hierarchical and balanced scorecard was proposed. This approach provides series of "instant snapshots" of the desired detail level that reflects the current situation in the company.

However, in order to actually organize a work process in the company, constantly evaluate the results, and adjust the development strategy of the company to changes, you need to answer some questions. How and where to take, or to measure the actual parameters, and how to relate assess their adequacy purposes with appropriate management processes, if necessary, to implement manages impact. In other words, a vicious management cycle with a feedback is needed (see Figure 1).

\section{Balance Scorecard}

The concept of Balanced Scorecard (BSC) was developed at the beginning of the 1990s by Robert Kaplan and David Norton from Harvard School of Economics. If the BSC was originally viewed as a tool for measuring and evaluating the effectiveness of the organization, in our time, it is a full-fledged methodology for strategic management.

Balanced Scorecard - is a description format of the organization for each of the four strategic aspects: internal business processes, development and training, customers, finance. 


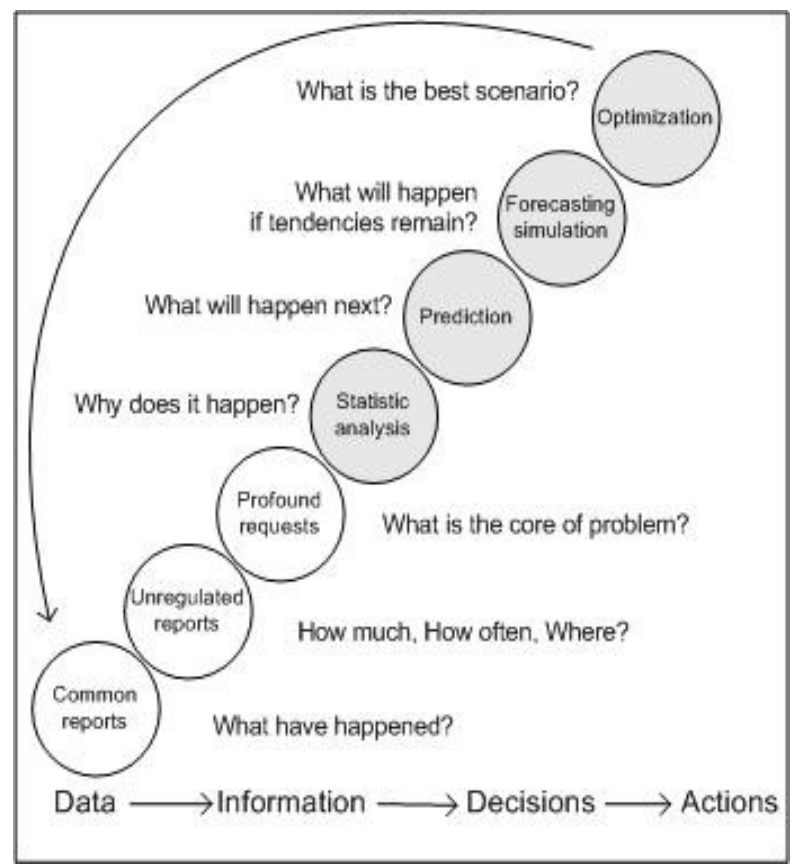

Figure 1

Each of the aspects contains main indicators for measuring the effectiveness of various processes. Another significant difference of BSC from its predecessors is the ability to organize treatment of intangible assets, all the more so in our time they play, of course, a great role [1].

Another advantage of the BSC is a special focus on the prospects of the company and their relationship with the current indicators. Correctly designed scorecard clearly shows a causal link between the current processes and prospects.

However, the BSC - is not a solution for all problems. Despite the fact that the system takes account of cause-and-effect relationships, it is very difficult to identify and build them on its basis. Moreover, a scorecard itself does not provide any assessments. Designing of the BSC cards - is a very laborious process, and adapting built maps to the processes of other companies is almost impossible.

However, BSC - is not a solution for all problems. Despite the fact that the system takes into account cause-and $\neg$ effect relationships, it is very difficult to identify and build them on its basis. Moreover, a scorecard itself does not provide any assessments. Designing of the BSC cards - is a very laborious process, and adapting built cards to the processes of other companies is almost impossible.

Despite of all the advantages of BSC, the methodology is more suitable for large scale simulation projects. If small and middle size projects are being developed it is more appropriate to use GoalQuestion-Metric (GQM) framework [2]. In that case organizational goals are identified, then questions are developed to determine whether the goals are being met, and then metrics are identified that can help answer the arising questions. The framework was developed at the University of Maryland as a mechanism for formalizing the tasks of characterization, planning, construction, analysis, learning, and feedback. The GQM paradigm was developed for all types of studies, particularly studies concerned with improvement issues.GQM consists of three primary steps [3]:

1. Generate a set of organizational goals.

2. Derive a set of questions relating to the goals.

3. Develop a set of metrics needed to answer the questions.

The goals are built according to the needs of the organization, and they help in determining whether or not organization was improved the way it wanted to.

The comparison of BSC and GQM can be found in [4].

\section{System Dynamics}

Modeling - is one of the ways to solve problems virtually rather than by working with them in the real world. Modeling is used in cases where experiments with real objects are impossible or too expensive. It includes a display of real-world problems in the world of models and subsequent analysis, and optimization models, finding solutions, and display solutions back to the real world. The popularity of system dynamics can be explained be the following advantages of the methodology [5]:

- the majority of real life complex systems with stochastic processes cannot be precisely described by analytically estimated mathematical models; that's why the modeling becomes the only available way to study their behavior.

- using the modeling techniques it is easier to provide much more effective control of experiment conditions than just experimenting with the system itself.

- modeling allows to study the long period of system operation in a short period of time;

J. Forrester, a professor at Massachusetts Institute of Technology, almost 50 years ago proposed a new idea of simulation, according to which complex systems should be modeled on the highest level of abstraction, when researcher abstracts from individual objects of the system and examine only the aggregate indicators of such objects, their interaction and interdependence in dynamics [6, 7]. Modeling paradigm where the systems are built researched charts causalities and global influence of some parameters on the other parameters over time, and then created on the basis of those diagrams on the computer model simulates, has been named system 
dynamics. In fact, this kind of modeling over all other paradigms to understand the essence of what is happening helps identify cause-effect relationships between objects and phenomena.

Despite such simplification, as aggregation, abstraction of individual characteristics, behavior and physical characteristics of the environment in which the process flow, system dynamics model proved quite productive for the study of many difficult problems. Models of business processes, city development, production and the dynamics of population, environment and development model of the epidemic are just some of the areas of simulation, which copes with the system dynamics.

Nowadays System Dynamics approach to simulation is widely used in the different areas of business $[8,9$, $10,11]$.

\section{Methodology}

The concept of the new methodology is based on the fusion of the two existing methodologies Balanced Scorecard (BSC) and System Dynamics (SD).

That kind of fusion in the proposed concept significantly improves both the process of model verification based on historical data and the process of strategic planning (as it is shown in [13] on SD and GQM fusion example).

Workflow - is a sequence of performed actions or a hierarchy of activity.

The Dynamic Enterprise Management principle of operation is implemented as a workflow concept, which includes the following stages of work with DEM projects .

Following the establishment of a new draft the analyst builds up an enterprise model "as is" in terms of system dynamics, then performs validation of the model according to the real-world data provided by the company: the model should be specified until the level of correctness becomes sufficient for the continuation of the work - checking all possible scenarios. Once the model is validated and enterprise development scenarios are set up, the expert chooses the best scenario (business plan) which meets specified business objectives. For example, the optimal scenario is the development scenario, in which the company remains resistant to the effects of the environment throughout its development process.

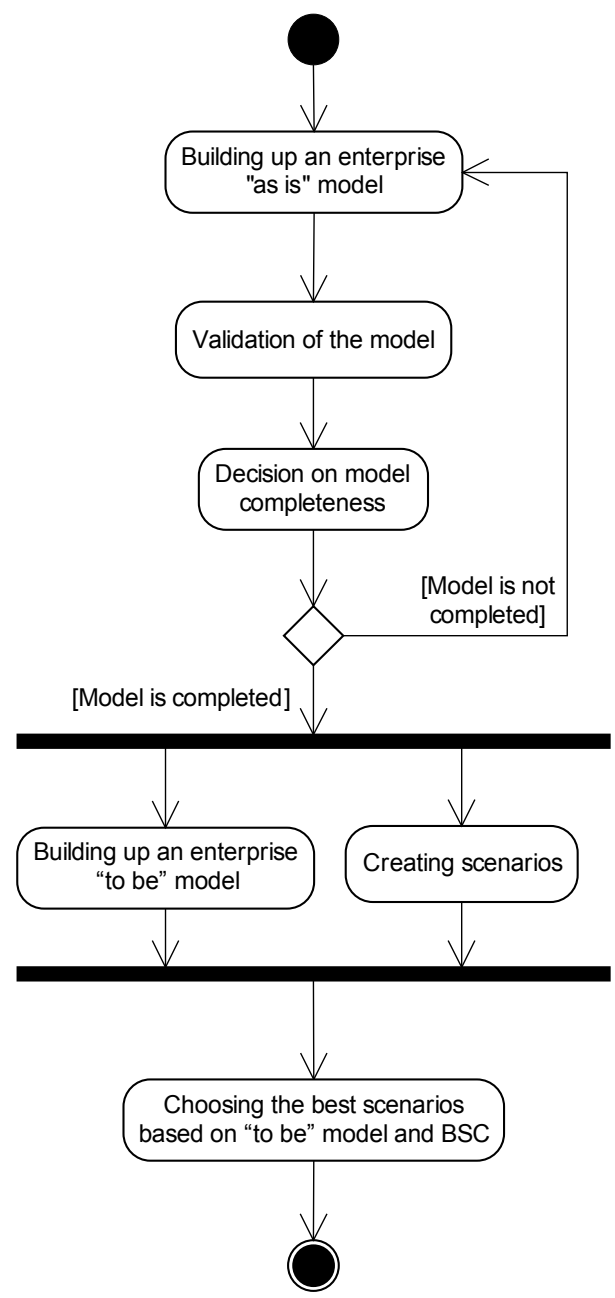

Figure 2. DEM Workflow

\section{DEM design}

According to the methodology described above, the information system (IS) DEM can be designed (figure 2).

System Dynamic Tool - is computer simulation software, where the structure of existing business processes of the company is built on; also different scenarios are simulated with the usage of a system dynamic tool.

OLAP (online analytical processing, analytical processing in real time) - is the technology of processing information, that also includes dynamic processes of drawing up and publishing records and documents. OLAP Technology serves, in particular, to prepare business records. Thus, the use of OLAP is necessary for the implementation of the software product Dynamic Enterprise Management.

Data Management layer includes Data Warehouse, integration buses and a set of protocols for the transfer of data from/to it. 


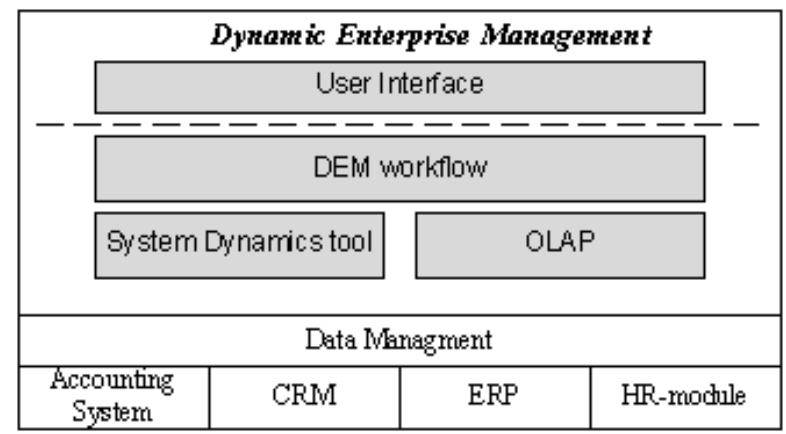

Figure 3. DEM design

In Data Warehouse all necessary information is gathered from various sources. Before data get to Data Warehouse, it should be properly processed. Enterprise databases of corporate systems, on the basis of which the Data Warehouse is built, will be called "transactional". Different departments may use different corporate information systems with their own transactional databases. Accordingly, before using these disparate data, they should be examined.

Here comes the question: how necessary information can be accumulated in Data Warehouse, that is how it is possible to transfer the data from different sources and different data formats. In order to be able to perform that kind of integration, a special integration bus is created, through which "communication" of data warehouse with a variety data sources of innovation is fulfilled.

As can be seen from Figure 2, at the bottom layer of DEM architecture there are many sources of data:

HR-module - is automated system staff management. Accounting System - a class of systems that perform accounting operations.

$C R M$ - Customer Relationship Management, System of Management Customer Relationship

ERP - Enterprise Resource Planning, systems of planning and company resource management.

\section{DEM prototype}

Currently, a prototype of DEM informational system is being developed system. The language of implementation is $\mathrm{C} \#$. As a platform for the prototype implementation the following Microsoft products and technologies were selected: Visual Studio. NET 2008, using the. NET Framework 3.5, MS SQL Server 2000, ASP. NET. Data integration is implemented using MS BizTalk product.

Three components were implemented:

- DB connector - is a component that provides a set of classes for connecting to the database DEM;

- Powersim connector - is a component that implements a set of classes for working with the simulation tool Powersim Studio;
- $1 C$ connector - is a component to connect to the database.

The prototype has a web-based user interface (implemented via ASP.NET).

During the prototyping phase it became clear that the development of scenario system planning should start with some certain project. Due to the rich knowledge in software engineering and business connections of the project team it was decided to develop DEM IS in application to strategic planning in software project management.

\section{Software Process Simulation}

There are some critical problems of strategic planning in the area of software development project management and in the area of project portfolio management: going out of time and money scope, closure of software development projects [12]

According to the research reports software companies are interested in the system of this kind. Furthermore to qualify for CMMI level 5 focusing on the process optimization is needed.

Simulation of software processes is the best approach here [13].

The number of simulation models for the software development processes exist $[13,14,15]$, the review of the majority of models is made it [13]. The latest tendencies are described in [16].

Attempts to the simulation for the software processes are described in [17]. However there is no composite system for strategic planning in software development.

\section{Conclusion}

In this work Dynamic Enterprise Management, an informational system for strategic planning, and its methodology were considered. DEM IS enables an expert (analyst) to estimate different development strategies, thus, to choose one, the most suitable strategy, from many. In our view, the system meets the modern, ever-changing business requirements. In addition, analysis of the existing strategic planning methodologies was carried out, and it showed that the DEM is a competitive system.

Implementation of the proposed system is realistic, as it is described in the architecture of IS Dynamic Enterprise Management. Currently prototype of DEM system is being developed within Software Engineering Department of Higher School of Economics. Some results have already been obtained in this field - the very first version of the prototype has been already developed. 


\section{References}

[1] R. S. Kaplan, D. P. Norton, "The Balanced Scorecard: Translating Strategy into Action", Harvard Business School Press, New York, 1996.

[2] Basili V R, "Software modeling and measurement: The goal/question/metric paradigm", Technical Report, CS-TR2956, Department of Computer Science, University of Maryland, College Park, MD 20742, September 1992.

[3] Basili, Victor; Gianluigi Caldiera, H. Dieter Rombach, "The Goal Question Metric Approach", 1994.

[4] L Buglione, A Abran, "Balanced scorecard and GQM: what are the differences?", Proceedings FESMA/AEMES Conference, 2000.

[5] Averill Law, W David Kelton, Simulation Modeling and Analysis. Third edition, McGraw-Hill.

[6] Jay Wright Forrester, Industrial Dynamics, Pegasus Communications, 1961.

[7] Jay Wright Forrester, Principles of Systems. Pegasus Communications, 1968

[8] Matthias Ruth, Bruce Hannon, Jay W. Forrester, Modeling Dynamic Economic Systems (Modeling Dynamic Systems), Springer; May 13, 1997
[9] John D. Sterman, Business Dynamics: Systems Thinking and Modeling for a Complex World, McGraw Hill Higher Education, December 1, 2000

[10] Bernard McGarvey, Bruce Hannon, Dynamic Modeling for Business Management: An Introduction (Modeling Dynamic Systems); 1 edition, Springer, January 8, 2004

[11] Kim Warren, Strategic Management Dynamics, Wiley, February 8, 2008

[12] Standish Group International Report, 2004

[13] Raymond J. Madachy, Software process dynamics, Wiley-interscience a john wiley \& sons, inc., publication ieee press, 2008

[14] Abdel-Hamid T. and Madnick S., Software Project Dynamics, Englewood Cliffs, NJ: Prentice-Hall, 1991.

[15] Acuna S., Juristo N., Moreno A., and Mon A., A Software Process Model Handbook for Incorporating People's Capabilities, 2005.

[16] Acuna S., Sanchez-Segura M., Series on Software Engineering and Knowledge Engineering Vol. 18. New Trends in Software Process Modeling, World Scientific Publishing, 2006.

[17] Seunghun Park, Hyeonjeong Kim, Dongwon Kang, Doo-Hwan Bae. Developing a Simulation Model Using a SPEM-Based Process Model and Analytical Models, 2008. 